\title{
新型双环新烟碱类似物的合成及其生物活性
}

\author{
陈寅波范杰夏爽程家高徐晓勇李忠* \\ (上海市化学生物学重点实验室 华东理工大学药学院 上海 200237)
}

\begin{abstract}
摘要 设计合成了六个新型双环新烟碱类似物, 通过 ${ }^{1} \mathrm{H} N \mathrm{NR},{ }^{13} \mathrm{C}$ NMR, 高分辨质谱和单晶结构对目标化合物的结构 进行了表征. 初步的生物活性测试表明: 在 $500 \mathrm{mg} \cdot \mathrm{L}^{-1}$ 浓度下，目标化合物 $\mathbf{6 a}, \mathbf{6 c} \sim \mathbf{6}$ 对豆蚜 (Aphis craccivora) 具有很 好的杀虫活性. 通过分子对接比较并解释了目标化合物 $\mathbf{6 a}$ 和吡虫啉的活性和作用模式的差异, 其结果表明目标化合物 6a 的吡啶环和受体残基色氨酸 147 的芳香环侧链具有 $\pi-\pi$ 堆积作用, 与吡虫啉的作用方式存在一定差异.

关键词 新烟碱类似物; 合成; 生物活性; 单晶结构; 分子对接
\end{abstract}

\section{Synthesis and Insecticidal Evaluation of Neonicotinoid Ana- logues with Bicyclic Ring System}

\author{
Chen, Yinbo Fan, Jie Xia, Shuang Cheng, Jiagao Xu, Xiaoyong Li, Zhong* \\ (Shanghai Key Laboratory of Chemical Biology, School of Pharmacy, East China University of \\ Science and Technology, Shanghai 200237)
}

\begin{abstract}
Six novel neonicotinoid analogues with bicyclic ring system were designed and synthesized, and their structures were characterized by ${ }^{1} \mathrm{H}$ NMR, ${ }^{13} \mathrm{C}$ NMR, HRMS and single crystal X-ray diffraction analysis. Preliminary bioassays showed that five target compounds $\mathbf{6 a}, \mathbf{6} \mathbf{c} \sim \mathbf{6} \mathbf{f}$ exhibited good insecticidal activities against cowpea aphids (Aphis craccivora) at 500 $\mathrm{mg} \bullet \mathrm{L}^{-1}$. Docking study was applied to investigate the effect of the bicyclic ring system of the target compound 6a on the biological activity, and compared with mode of action of imidacloprid (IMI) and compound 6a. It was found that the only $\pi-\pi$ stacking interaction was found between the plane of pyridine of the target compounds $\mathbf{6 a}$ and the aromatic side chain of Tyr 147, which was different binding mode with that of IMI.
\end{abstract}

Keywords neonicotinoid analogue; synthesis; bioactivity; crystal structure; molecular docking

当今，以吡虫啉为代表的新烟碱类杀虫剂是继有机 磷、有机氯、氨基甲酸酯及拟除虫菊酯后具有里程碑意 义的新一代杀虫剂. 新烟碱类杀虫剂具有结构新颖、作 用机制独特、杀虫谱广、对哺乳动物和各类水生生物安 全、环境友好以及良好的内吸性和传导性等突出优点, 已经成功应用于农业害虫防治、动物健康保护和卫生害 虫防治等各个领域 ${ }^{[1 ~ 10]}$.

目前, 已商品化的新烟碱类杀虫剂有 7 个品种, 根 据结构特性主要分为两类: 开环化合物和闭环化合物. 闭环化合物中, 包括五元环结构的吡虫啉以及噻虫啉 (德国拜耳公司)和六元环结构的噻虫嗪(瑞士先正达公
司). 然而, 双环结构的新烟碱类杀虫剂的活性研究很 少被报道 ${ }^{[11 ~ 13]}$.

继三种经典的新烟碱类化合物与烟碱乙酰胆碱受 体(nicotinic acetylcholine receptor, nAChR)的作用模式 分别由 Yamamoto, Kagabu 和 Casida 等 ${ }^{[14 \sim 16]}$ 提出之后, 2007 年, 钱旭红等 ${ }^{[17]}$ 提出了氢键协同的 $\pi-\pi$ 堆积作用机 制, 该机制的提出为基于靶标的新烟碱活性分子的设计 提出了新的依据. 2008 年, Casida 和 Sattelle 等 ${ }^{[18,19]}$ 分别 得到了蜗牛乙酰胆碱结合蛋白 (acetylcholine binding protein, AChBP)与吡虫啉的复合物的晶体结构, 均可以 发现: 一方面硝基上的氧原子朝向氨基酸残基形成氢键

\footnotetext{
*E-mail: lizhong@ecust.edu.cn

Received August 26, 2013; revised September 25, 2013; published online October 28, 2013.

Project supported by the National Basic Research Program of China (973 Program, No. 2010CB126100), the National High Technology Research and Development Program of China (863 Program, No. 2011AA10A207), the National Key Technology R\&D Program of China (No. 2011BAE06B01) and the National Natural Science Foundation of China (No. 21272071).

国家重点基础研究发展计划(973 计划, No. 2010CB126100)、国家高技术研究发展计划(863 计划, No. 2011AA10A207)、“十一五” 国家科技支撑计划 (No. 2011BAE06B01) 和国家自然科学基金(No. 21272071)资助项目.
} 
作用; 另一方面, 吡虫啉结构上的硝基胍平面与蛋白结 构中的酪氨酸产生 $\pi-\pi$ 堆积作用. 在氢键协同参与的 $\pi-\pi$ 堆积作用下, 新烟碱类化合物表现出了显著的杀虫活 性，该实验结论与钱旭红等理论计算提出的新作用模式 是相一致的. 同时, 本研究组根据上述的分子设计的理 论设计合成了一系列的新烟碱类似物, 化合物 $\mathbf{1}$ 和 $\mathbf{2}$ 的 杀虫活性都优于吡虫啉 ${ }^{[20 ~ 22]}$. 但是, 化合物 1 的不稳定 性限制其商品化, 本研究组在化合物 $\mathbf{1}$ 的基础上继续做 进一步的结构修饰(图 1), 希望提高其稳定性.

本文设计合成了六个新型双环新烟碱类似物, 目的 在于提高化合物 1 的稳定性和 $\pi-\pi$ 堆积作用特性. 目标 化合物的合成路线如 Scheme 1 所示. 通过 ${ }^{1} \mathrm{H}$ NMR, ${ }^{13} \mathrm{C}$ NMR 和高分辨质谱对目标化合物的结构进行表征, 获 得了目标化合物 $6 \mathrm{a}$ 的单晶结构, 对目标化合物 $\mathbf{6 a} \sim \mathbf{6 f}$ 进行了初步的活性测试, 并进行了分子对接的研究.

\section{1 实验部分}

\section{1 仪器与试剂}

熔点仪为瑞士 Büchi 公司生产的 B540型熔点仪，未 经校正; Bruker AM-400 (400 MHz)核磁共振仪, 以 $\mathrm{CDCl}_{3}$ 或 DMSO- $d_{6}$ 为溶剂, TMS 为内标; 柱层析采用柱 层析硅胶(200 300 目). 所用的试剂均为市售分析纯.

\section{2 合成}

1.2.1 $N^{1}$-[(6-氯吡啶-3-基)甲基]苯-1,2-二胺(4)的合成

室温条件下，将 2-氯-5-氯甲基吡啶 $(3,1.0 \mathrm{mmol})$ 溶解在 $5 \mathrm{~mL}$ 乙腈中, 缓慢滴加到邻苯二胺 $(2.0 \mathrm{mmol})$, 碳酸钾 $(1.2 \mathrm{mmol})$ 的乙腈 $(5 \mathrm{~mL})$ 混合液中, 滴加完毕, 升 高温度至 $60{ }^{\circ} \mathrm{C}$, 继续反应 $4 \mathrm{~h}$, 反应完毕, 旋干溶剂, 用二氯甲烷稀释，用水萃取，收集有机相，用无水硫酸 钠干燥，抽滤，浓缩反应液，用石油醚与乙酸乙酯为淋

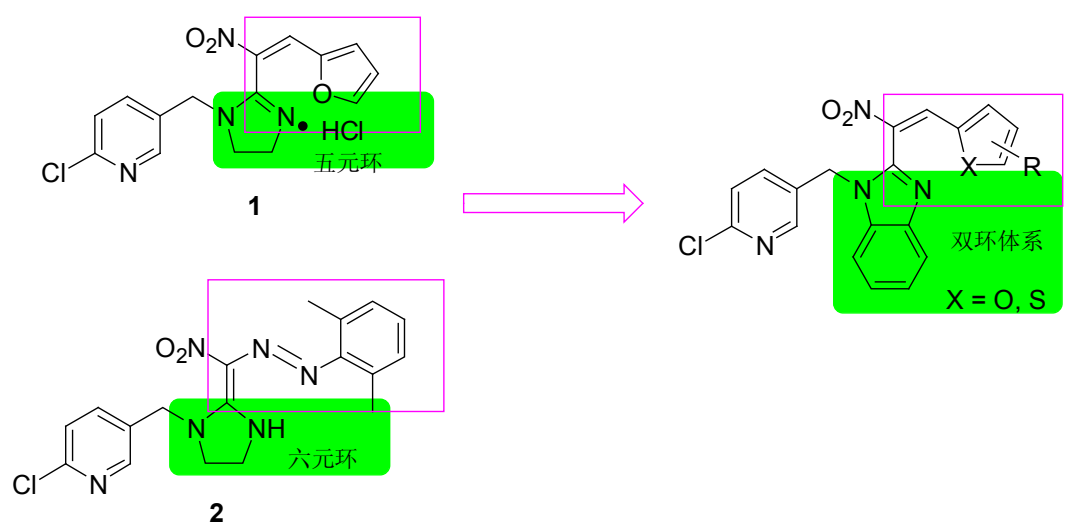

图 1 目标化合物的分子设计

Figure 1 Molecular design of the neonicotinoid analogues

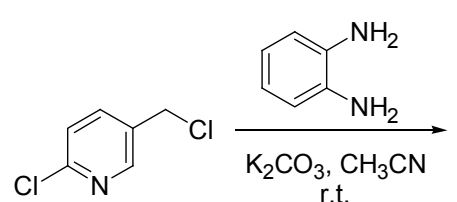

3

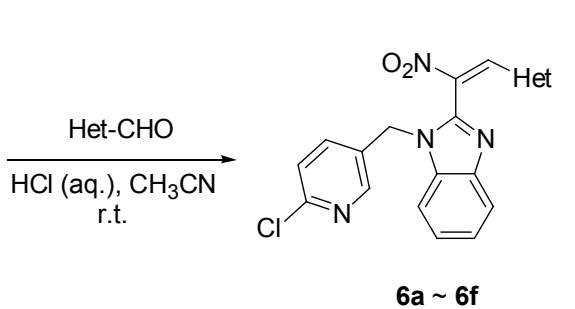<smiles>CC(C)=C[N+](=O)[O-]</smiles><smiles>O=[N+]([O-])/C=C1\Nc2ccccc2N1Cc1ccc(Cl)nc1</smiles>

5

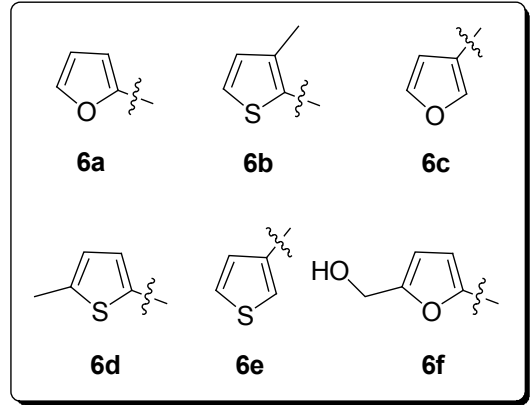

Scheme 1 
洗液, 通过硅胶柱层析分离得到目标化合物 $\mathbf{4}$, 为棕色 液体, 产率 $50 \% .{ }^{1} \mathrm{H}$ NMR $\left(400 \mathrm{MHz}, \mathrm{DMSO}-d_{6}\right) \delta: 8.44$ (s, 1H), $7.81(\mathrm{~d}, J=8.0 \mathrm{~Hz}, 1 \mathrm{H}), 7.44(\mathrm{~d}, J=8.0 \mathrm{~Hz}, 1 \mathrm{H})$, $6.63(\mathrm{t}, J=4.4 \mathrm{~Hz}, 1 \mathrm{H}), 6.48 \sim 6.46(\mathrm{~m}, 2 \mathrm{H}), 6.38(\mathrm{t}, J=$ $4.4 \mathrm{~Hz}, 1 \mathrm{H}), 5.19$ (t, $J=4.8 \mathrm{~Hz}, 1 \mathrm{H}), 4.59$ (bs, 2H), 4.34 $(\mathrm{d}, J=4.8 \mathrm{~Hz}, 2 \mathrm{H}) ;{ }^{13} \mathrm{C}$ NMR $\left(100 \mathrm{MHz}\right.$, DMSO- $\left.d_{6}\right) \delta$ : $149.5,149.1,139.3,136.0,135.9,135.4,124.3,118.1$, 118.0, 114.9, 111.1, 44.2 .

1.2.2 (E)-1-[(6-氯吡啶-3-基) 甲基]-2-(硝基亚甲 基)-2,3-二氢(- $1 H$-苯并 $[d]$ 味唑(5)的合成

向 $25 \mathrm{~mL}$ 的单口烧瓶中加入中间体 4 (1.0 mmol), 1,1-二硫甲基-2-硝基乙烯(1.0 mmol)和 $5 \mathrm{~mL}$ 乙醇, 加热 回流 $12 \mathrm{~h}$. 反应结束后冷却反应液, 固体析出, 过滤, 用少量乙醇洗涤滤饼, 干燥得到目标化合物 $\mathbf{5}$, 黄色固 体, 产率 $80 \%$. m.p. $183 \sim 184{ }^{\circ} \mathrm{C} ;{ }^{1} \mathrm{H}$ NMR $(400 \mathrm{MHz}$, DMSO- $\left.d_{6}\right) \delta: 8.43(\mathrm{~d}, J=2.4 \mathrm{~Hz}, 1 \mathrm{H}), 7.67 \sim 7.61(\mathrm{~m}, 3 \mathrm{H})$, $7.49(\mathrm{~d}, J=8.4 \mathrm{~Hz}, 1 \mathrm{H}), 7.32 \sim 7.26(\mathrm{~m}, 3 \mathrm{H}), 5.49(\mathrm{~s}, 2 \mathrm{H})$, $3.37(\mathrm{~s}, 1 \mathrm{H}) ;{ }^{13} \mathrm{C}$ NMR (100 MHz, DMSO- $\left.d_{6}\right) \delta: 150.3$, $149.2,149.0,147.4,139.0,131.9,131.3,130.6,125.0$, $124.3,124.2,113.2,110.4,43.2$.

\subsection{3 目标化合物 $\mathbf{6 a} \sim \mathbf{6} \mathbf{f}$ 合成通法}

将中间体 $5(1.0 \mathrm{mmol}), 3 \mathrm{~mL}$ 无水乙腈, 五元杂环 醛 $(1.2 \mathrm{mmol}$ ), 催化量的盐酸依次加入到 $25 \mathrm{~mL}$ 的单口 烧瓶中, 室温下搅拌, TLC 监控反应, 反应结束, 旋干溶 剂, 反应液加入饱和碳酸氢钠的溶液调节 $\mathrm{pH}$ 值为 8 左 右, 二氯甲烷萃取, 收集有机相, 用无水硫酸钠干燥, 抽滤, 浓缩反应液, 用石油醚与乙酸乙酯为淋洗液, 通 过硅胶柱层析分离得到目标化合物 $\mathbf{6 a} \sim \mathbf{6 f}$.

(E)-1-[(6-氯吡啶-3-基)甲基]-2-[2-(呋喃-2-基)-1-硝 基乙烯基]- $1 H$-苯并 $[d]$ 咪唑(6a): 黄色固体, 收率 78\%. m.p. $183 \sim 184{ }^{\circ} \mathrm{C} ;{ }^{1} \mathrm{H}$ NMR $\left(400 \mathrm{MHz}, \mathrm{CDCl}_{3}\right) \delta: 8.40$ (s, $1 \mathrm{H}), 8.26(\mathrm{~d}, J=2.4 \mathrm{~Hz}, 1 \mathrm{H}), 7.95 \sim 7.92(\mathrm{~m}, 1 \mathrm{H}), 7.44 \sim$ $7.38(\mathrm{~m}, 3 \mathrm{H}), 7.35$ (dd, $J=8.4,2.4 \mathrm{~Hz}, 1 \mathrm{H}), 7.33 \sim 7.30$ (m, 1H), 7.15 (d, $J=8.4 \mathrm{~Hz}, 1 \mathrm{H}), 6.70(\mathrm{~d}, J=3.6 \mathrm{~Hz}, 1 \mathrm{H})$, 6.49 (dd, $J=3.6,1.6 \mathrm{~Hz}, 1 \mathrm{H}), 5.26$ (s, 2H); ${ }^{13} \mathrm{C}$ NMR $(100$ $\left.\mathrm{MHz}, \mathrm{CDCl}_{3}\right) \delta: 151.5,149.1,148.3,145.8,143.2,142.9$, $137.6,134.9,134.6,129.7,128.4,124.7,124.4,123.4$, 123.3, 121.4, 113.9, 110.4, 45.4; HRMS (EI+) calcd for $\mathrm{C}_{19} \mathrm{H}_{13} \mathrm{~N}_{4} \mathrm{O}_{3}{ }^{35} \mathrm{Cl}$ 380.0676, found 380.0678; calcd for $\mathrm{C}_{19} \mathrm{H}_{13} \mathrm{~N}_{4} \mathrm{O}_{3}{ }^{37} \mathrm{Cl} 382.0647$, found 382.0668 .

(E)-1-[(6-氯吡啶-3-基)甲基]-2-[2-(3-甲基噻吩-2基)-1-硝基乙烯基]- $1 H$-苯并 $[d]$ 咪唑(6b): 黄色固体, 收 率 70\%. m.p. 179 180 ${ }^{\circ} \mathrm{C} ;{ }^{1} \mathrm{H}$ NMR (400 MHz, $\mathrm{CDCl}_{3}$ ) $\delta: 8.86(\mathrm{~s}, 1 \mathrm{H}), 8.31(\mathrm{~s}, 1 \mathrm{H}), 7.97(\mathrm{~d}, J=8.0 \mathrm{~Hz}, 1 \mathrm{H})$, $7.46 \sim 7.38(\mathrm{~m}, 4 \mathrm{H}), 7.33 \sim 7.31(\mathrm{~m}, 1 \mathrm{H}), 7.13(\mathrm{~d}, J=8.0$
$\mathrm{Hz}, 1 \mathrm{H}), 6.94$ (d, $J=5.2 \mathrm{~Hz}, 1 \mathrm{H}), 5.24$ (s, 2H), 2.50 (s, $3 \mathrm{H}) ;{ }^{13} \mathrm{C}$ NMR $\left(100 \mathrm{MHz}, \mathrm{CDCl}_{3}\right) \delta: 151.5,150.0,148.5$, $143.4,142.1,137.9,135.1,134.9,134.2,134.1,131.2$, 129.4, 127.8, 125.0, 124.4, 123.5, 121.6, 110.6, 45.4, 15.0; HRMS (EI+) calcd for $\mathrm{C}_{20} \mathrm{H}_{15} \mathrm{~N}_{4} \mathrm{O}_{2} \mathrm{~S}^{35} \mathrm{Cl} 410.0604$, found 410.0601; calcd for $\mathrm{C}_{20} \mathrm{H}_{15} \mathrm{~N}_{4} \mathrm{O}_{2} \mathrm{~S}^{37} \mathrm{Cl} 412.0575$, found 412.0576 .

(E)-1-[(6-氯吡啶-3-基)甲基]-2-[2-(呋喃-3-基)-1-硝 基乙烯基]-1H-苯并 $[d]$ 咪唑(6c)：黄色固体，收率 74\%. m.p. $169 \sim 170{ }^{\circ} \mathrm{C} ;{ }^{1} \mathrm{H}$ NMR $\left(400 \mathrm{MHz}, \mathrm{CDCl}_{3}\right) \delta: 8.51$ (s, $1 \mathrm{H}), 8.26(\mathrm{~d}, J=2.4 \mathrm{~Hz}, 1 \mathrm{H}), 7.96 \sim 7.94(\mathrm{~m}, 1 \mathrm{H}), 7.78(\mathrm{~s}$, $1 \mathrm{H}), 7.46 \sim 7.39(\mathrm{~m}, 2 \mathrm{H}), 7.34 \sim 7.30(\mathrm{~m}, 2 \mathrm{H}), 7.28 \sim 7.27$ (m, $1 \mathrm{H}), 7.16(\mathrm{~d}, J=8.0 \mathrm{~Hz}, 1 \mathrm{H}), 5.42(\mathrm{~d}, J=1.6 \mathrm{~Hz}, 1 \mathrm{H})$, $5.23(\mathrm{~s}, 2 \mathrm{H}) ;{ }^{13} \mathrm{C}$ NMR $\left(100 \mathrm{MHz}, \mathrm{CDCl}_{3}\right) \delta: 151.6,149.8$, $148.4,145.6,142.9,142.6,137.6,137.4,134.5,133.5$, $129.5,125.1,124.5,123.6,121.5,117.9,110.5,108.2$, 45.4. HRMS (EI+) calcd for $\mathrm{C}_{19} \mathrm{H}_{13} \mathrm{~N}_{4} \mathrm{O}_{3}{ }^{35} \mathrm{Cl} 380.0676$, found 380.0651; calcd for $\mathrm{C}_{19} \mathrm{H}_{13} \mathrm{~N}_{4} \mathrm{O}_{3}{ }^{37} \mathrm{Cl} 382.0647$, found 382.0674 .

(E)-1-[(6-氯吡啶-3-基)甲基]-2-[2-(5-甲基噻吩-2基)-1-硝基乙烯基]- $1 H$-苯并 $[d]$ 味唑(6d)：黄色固体，收 率 68\%. m.p. 182 184 ${ }^{\circ} \mathrm{C} ;{ }^{1} \mathrm{H}$ NMR $\left(400 \mathrm{MHz}, \mathrm{CDCl}_{3}\right)$ $\delta: 8.72(\mathrm{~s}, 1 \mathrm{H}), 8.31(\mathrm{~d}, J=2.4 \mathrm{~Hz}, 1 \mathrm{H}), 7.99 \sim 7.96(\mathrm{~m}$, $1 \mathrm{H}), 7.46 \sim 7.38(\mathrm{~m}, 3 \mathrm{H}), 7.33 \sim 7.31(\mathrm{~m}, 2 \mathrm{H}), 7.15$ (d, $J=$ $8.4 \mathrm{~Hz}, 1 \mathrm{H}), 6.79(\mathrm{~d}, J=3.6 \mathrm{~Hz}, 1 \mathrm{H}), 5.24(\mathrm{~s}, 2 \mathrm{H}), 2.38(\mathrm{~s}$, $3 \mathrm{H}) ;{ }^{13} \mathrm{C}$ NMR $\left(100 \mathrm{MHz}, \mathrm{CDCl}_{3}\right) \delta: 153.4,151.5,148.5$, $143.4,142.2,140.2,137.9,136.3,134.8,133.8,131.2$, 129.5, 127.5, 124.9, 124.4, 123.4, 121.6, 110.6, 45.6, 16.2; HRMS (EI+) calcd for $\mathrm{C}_{20} \mathrm{H}_{15} \mathrm{~N}_{4} \mathrm{O}_{2} \mathrm{~S}^{35} \mathrm{Cl} 410.0604$, found 410.0593; calcd for $\mathrm{C}_{20} \mathrm{H}_{15} \mathrm{~N}_{4} \mathrm{O}_{2} \mathrm{~S}^{37} \mathrm{Cl} 412.0575$, found 412.0597 .

(E)-1-[(6-氯吡啶-3-基)甲基]-2-[1-硝基-2-(噻吩-3基)乙烯基]- $1 H$-苯并 $[d]$ 咪唑(6e)：黄色固体，收率 $66 \%$. m.p. $176 \sim 178{ }^{\circ} \mathrm{C} ;{ }^{1} \mathrm{H}$ NMR (400 MHz, $\mathrm{CDCl}_{3}$ ) $\delta: 8.57$ (s, $1 \mathrm{H}), 8.21(\mathrm{~d}, J=2.0 \mathrm{~Hz}, 1 \mathrm{H}), 7.96 \sim 7.94(\mathrm{~m}, 1 \mathrm{H}), 7.55(\mathrm{~d}$, $J=2.0 \mathrm{~Hz}, 1 \mathrm{H}), 7.45 \sim 7.40(\mathrm{~m}, 2 \mathrm{H}), 7.35 \sim 7.32(\mathrm{~m}, 1 \mathrm{H})$, $7.23(\mathrm{dd}, J=8.4,2.4 \mathrm{~Hz}, 1 \mathrm{H}), 7.17(\mathrm{dd}, J=5.2,2.8 \mathrm{~Hz}$, $1 \mathrm{H}), 7.06(\mathrm{~d}, J=8.0 \mathrm{~Hz}, 1 \mathrm{H}), 6.32(\mathrm{~d}, J=5.2 \mathrm{~Hz}, 1 \mathrm{H}), 5.20$ (s, $2 \mathrm{H}) ;{ }^{13} \mathrm{C}$ NMR $\left(100 \mathrm{MHz}, \mathrm{CDCl}_{3}\right) \delta: 151.5,148.3$, $143.1,142.8,137.5,137.1,135.8,135.7,134.6,131.5$, $129.5,128.1,127.0,125.0,124.4,123.6,121.5,110.5$, 45.3; HRMS (EI+) calcd for $\mathrm{C}_{19} \mathrm{H}_{13} \mathrm{~N}_{4} \mathrm{O}_{2} \mathrm{~S}^{35} \mathrm{Cl} 396.0448$, found 396.0446; calcd for $\mathrm{C}_{19} \mathrm{H}_{13} \mathrm{~N}_{4} \mathrm{O}_{2} \mathrm{~S}^{37} \mathrm{Cl} 398.0418$, found 398.0432 .

(E)-(5-(2-(1-((6-氯吡啶-3-基) 甲基)-1H-苯并 $[d]$ 咪 
唑-2-基)-2-硝基乙烯基)呋喃-2-基)甲醇(6f)：黄色固体, 收率 68\%. m.p. 189 191 ${ }^{\circ} \mathrm{C} ;{ }^{1} \mathrm{H}$ NMR $(400 \mathrm{MHz}$, $\left.\mathrm{CDCl}_{3}\right) \delta: 8.29(\mathrm{~s}, 1 \mathrm{H}), 8.11(\mathrm{~s}, 1 \mathrm{H}), 7.93(\mathrm{q}, J=2.8 \mathrm{~Hz}$ $1 \mathrm{H}), 7.47 \sim 7.39(\mathrm{~m}, 3 \mathrm{H}), 7.36 \sim 7.34(\mathrm{~m}, 1 \mathrm{H}), 7.18(\mathrm{~d}, J=$ $8.4 \mathrm{~Hz}, 1 \mathrm{H}), 6.70(\mathrm{~d}, J=2.4 \mathrm{~Hz}, 1 \mathrm{H}), 6.38(\mathrm{~d}, J=3.2 \mathrm{~Hz}$, 1H), 5.28 (s, 2H), 4.29 (s, 2H), 2.63 (bs, $1 \mathrm{H}) ;{ }^{13} \mathrm{C} \mathrm{NMR}$ $\left(100 \mathrm{MHz}, \mathrm{CDCl}_{3}\right) \delta: 161.8,151.3,148.2,145.0,142.9$, $142.8,137.6,134.5,134.3,129.9,128.4,125.0,124.9$, 124.4, 123.6, 121.1, 111.5, 110.2, 57.2, 45.3; HRMS (EI + ) calcd for $\mathrm{C}_{20} \mathrm{H}_{15} \mathrm{~N}_{4} \mathrm{O}_{4}{ }^{35} \mathrm{Cl}$ 410.0782, found 410.0785; calcd for $\mathrm{C}_{20} \mathrm{H}_{15} \mathrm{~N}_{4} \mathrm{O}_{4}{ }^{37} \mathrm{Cl} 412.0752$, found 412.0773 .

\section{3 生物活性测定}

以豆蚜 (Aphis craccivora) 为测试对象, 采用浸渍法 测试. 准确称量各样品, 化合物溶解于 DMSO 和稀释的 Triton X-100 $\left(0.1 \mathrm{mg} \cdot \mathrm{L}^{-1}\right)$ 的水中, 配制浓度为 500 和 $250 \mathrm{mg} \cdot \mathrm{L}^{-1}$. 空白药由 DMSO 和稀释的 Triton X-100 $\left(0.1 \mathrm{mg} \cdot \mathrm{L}^{-1}\right)$ 的水配制而成. 将附有一定数量的待测试 虫的虫豆叶在药液中浸泡 $5 \mathrm{~s}$, 取出晾干, 和试虫一并放 入干净的器典中, 置于恒温恢复室内, $48 \mathrm{~h}$ 后检查试虫 的死亡情况. 每个实验重复三次, 结果取平均值. 以吡 虫啉为对照药剂在相同的条件下测定其生物活性.

\section{4 分子对接方法}

从 PDB 库(http://www.rcsb.org)下载 Aplysia californica AChBP (Ac-AChBP) 与吡虫啉复合晶体(编号 $3 \mathrm{C} 79$, 分辨率为 $2.48 \AA$ ) 的 PDB 文件. 蛋白结构的预处 理在 Maestro 7.5 中的 Protein Preparation Wizard 模块进 行, 修正结构中不合理的化学键, 对蛋白进行去水、加 氢及加电荷. 再利用 Maestro 中 Ligprep 模块对目标化合 物 6a 进行结构优化, 采用 OPLS-2005 力场计算较低能 量的分子三维结构和 $\mathrm{pH} 5 \sim 9$ 时小分子可能的质子化状 态, 使化合物具有正确的手性、离子状态、合理的键长 键角. 采用 GOLD v3.2 软件(The Cambridge Crystallographic Data Centre, Cambridge, U.K.)进行分子对接, 将 $3 C 79$ 晶体结构中吡虫啉与蛋白结合位点周围 $10 \AA$ 内的 原子定义为口袋位置, 采用 GoldScore 打分函数, 输出 20 个对接结果, 选取打分最高的对接构象进行分析.

\section{2 结果与讨论}

\section{1 目标化合物 X 衍射数据收集与结构鉴定}

目标化合物 6a 从乙醚和丙酮中培养得到黄色晶体. 选取尺寸为 $0.386 \mathrm{~mm} \times 0.369 \mathrm{~mm} \times 0.235 \mathrm{~mm}$ 的黄色晶 体放置在 $X$ 射线单晶衍射仪上, 测定温度为 $20{ }^{\circ} \mathrm{C}$, 由 图 2 所示, 晶体结构分析如下, 目标化合物 $6 \mathbf{a}$ 为单斜 晶体, 晶胞参数为 $a=8.8305(9) \AA, b=20.802(2) \AA, c=$
9.9504(10) $\AA, \alpha=90^{\circ}, \beta=112.852(2)^{\circ}, \gamma=90^{\circ}, \mu=0.257$ $\mathrm{mm}^{-1}, V=1684.3(3) \AA^{3}, Z=4, D_{\mathrm{x}}=1.502 \mathrm{Mg} \cdot \mathrm{m}^{-3}$, $F(000)=784,2.43^{\circ} \leqslant \theta \leqslant 26.00^{\circ}$. 目标化合物 $\mathbf{6 a}$ 可以通 过单晶衍射来确认其结构, 而化合物 $\mathbf{1}$ 由于其不稳定性 无法得到单晶结构, 其在溶剂就会自身发生 Diels-Alder 反应得到六氢咪唑并 $[1,2-\mathrm{a}]$ 吡啶衍生物 ${ }^{[23]}$. 而本工作中, 目标化合物 6a 在溶剂中很稳定, 可以得到单晶结构, 间 接说明通过引入双环结构来增加化合物 $\mathbf{1}$ 的稳定性是一 种合理的分子设计.

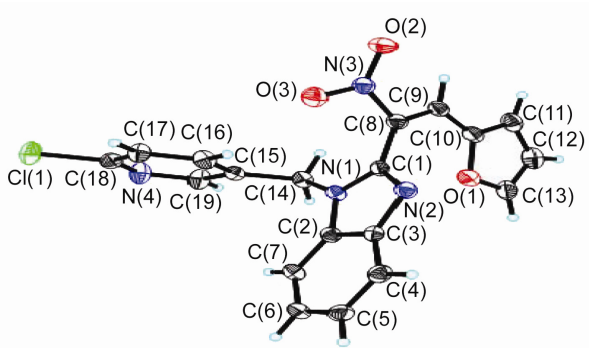

图 2 目标化合物 $6 \mathrm{a}$ 的分子结构图

Figure 2 Molecular structure of compound 6a

\section{2 生物活性}

由表 1 可知, 在 $500 \mathrm{mg} \cdot \mathrm{L}^{-1}$ 浓度下, 目标化合物 6a, 6c 6f 对蚜虫的杀虫活性为 $50 \% \sim 100 \%$; 当浓度降低 到 $250 \mathrm{mg} \cdot \mathrm{L}^{-1}$, 目标化合物 $6 \mathbf{e}$ 和 $6 \mathbf{f}$ 对蚜虫的杀虫活性 分别为 $72.9 \%$ 和 $42.6 \%$. 其结果表明, 杂环取代基为噻 吩和呋喃时, 活性差异影响不大. 但是, 当噻吩的 3-位 引入甲基时，目标化合物 $\mathbf{6 b}$ 的活性就大大降低.

表 1 目标化合物 $\mathbf{6 a} \sim \mathbf{6 f}$ 和吡虫啉对蚜虫的杀虫活性 Table 1 Insecticidal activities of compounds $\mathbf{6 a} \sim \mathbf{6} \mathbf{f}$ and imidacloprid against pea aphids

\begin{tabular}{ccc}
\hline 化合物 & \multicolumn{2}{c}{ 死亡率/\% } \\
\hline $\mathbf{6 a}$ & $500 \mathrm{mg} \cdot \mathrm{L}^{-1}$ & $250 \mathrm{mg} \cdot \mathrm{L}^{-1}$ \\
$\mathbf{6 b}$ & 50.0 & N.t. ${ }^{a}$ \\
$\mathbf{6 c}$ & 7.9 & N.t. \\
$\mathbf{6 d}$ & 70.9 & N.t. \\
$\mathbf{6 e}$ & 65.5 & N.t. \\
$\mathbf{6 f}$ & 100 & 72.9 \\
吡虫啉 & 82.8 & 42.6 \\
${ }^{a} \mathrm{~N}$ 米测试 & 100 & 100 \\
\hline
\end{tabular}

\section{3 分子对接}

至今, 昆虫的 nAChR 复合物晶体未有报道, 因此, 本工作采用结合吡虫啉的 Ac-AChBP 的复合物晶体结 构作为受体 ${ }^{[10,24,25]}$. 如图 3 所示, 图 3a 为目标化合物 6a 与 $\mathrm{AChBP}$ 的相互作用, 图 $3 \mathrm{~b}$ 为目标化合物 $\mathbf{6 a}$ 与 $\mathrm{AChBP}$ 晶体结构中吡虫啉叠合图. 其中, 目标化合物 6a 的吡啶环可与色氨酸 147 的芳环形成 $\pi-\pi$ 堆积相互作 用, 而吡虫啉的咪唑环与酪氨酸 188 的芳环形成 $\pi-\pi$ 作 
用，与目标化合物 6a 的作用残基不同. 此前的报道中 [26]，水分子可以作为 “桥梁” 使吡虫啉吡啶环上的氮原 子与异亮氨酸 118 的氮原子和异亮氨酸 106 的氧原子形 成氢键网络, 吡虫啉硝基氧原子可与半胱氨酸 190 的氮 原子形成氢键作用. 而这些作用在目标化合物 6a 与 $\mathrm{AChBP}$ 的作用中均未发现, 因此, 目标化合物 $\mathbf{6 a}$ 与吡 虫啉的作用方式可能存在一定差异.

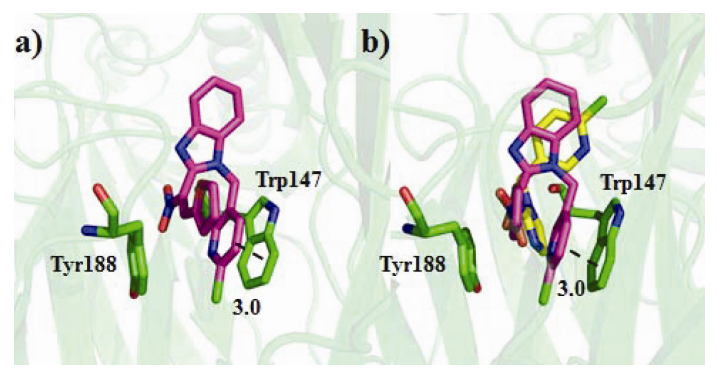

图 3 (a)目标化合物 6a 与 AChBP 的相互作用和(b)目标化合 物 $6 \mathbf{a}$ 与 $\mathrm{AChBP}$ 晶体结构中吡虫啉叠合图

Figure 3 (a) The predictive binding site interaction of compound 6a with AChBP and (b) the conformational comparison of 6a and IMI as observed in the AChBP binding pocket.

\section{3 结论}

通过三步反应合成了六个新型双环新烟碱类似物, 其结构通过 ${ }^{1} \mathrm{H} N M R,{ }^{13} \mathrm{C}$ NMR, 高分辨质谱和单晶结构 进行表征. 初步的生物活性测试表明: 在 $500 \mathrm{mg} \cdot \mathrm{L}^{-1}$ 浓 度下, 目标化合物 $\mathbf{6 a}, \mathbf{6 c} \sim \mathbf{6 f}$ 对豆䖮(Aphis craccivora) 具 有很好的杀虫活性. 单晶结构表明: 引入双环结构有利 于增加化合物 1 的稳定性. 分子对接的结果表明: 目标 化合物 6a 与吡虫啉的作用方式存在一定差异, 引入双 环结构导致构型的变化, 这也是目标化合物 $\mathbf{6 a}$ 活性大 大降低的可能的原因之一.

\section{References}

[1] Jeschke, P.; Nauen, R. Pest. Manage. Sci. 2008, 64, 1084.

[2] Kagabu, S. J. Agric. Food Chem. 2011, 59, 2887.

[3] Jeschke, P.; Nauen, R.; Schindler, M.; Elbert, A. J. Agric. Food Chem. 2011, 59, 2897.

[4] Tomizawa, M.; Kagabu, S.; Casida, J. E. J. Agric. Food Chem.
2011, 59, 2918.

[5] Shao, X. S.; Lee, P. W.; Liu, Z. W.; Xu, X. X.; Li, Z.; Qian, X. H. J. Agric. Food Chem. 2011, 59, 2943.

[6] Tomizawa, M.; Maltby, D.; Talley, T. T.; Durkin, K. A.; Medzihradszky, K. F.; Burlingame, A. L.; Taylor, P.; Casida, J. E. Proc. Natl. Acad. Sci. U. S. A. 2008, 105, 1728.

[7] Matsuda, K.; Shimomura, M.; Ihara, M.; Akamatsu, M.; Sattelle, D. B. Biosci. Biotechnol. Biochem. 2005, 69, 1442.

[8] Tomizawa, M.; Casida, J. E. Annu. Rev. Pharmacol. Toxicol. 2005 , $45,247$.

[9] Xue, S. J.; Bu, H. F.; Liu, L.; Xu, X.; Ma, X. B. Chin. J. Chem. 2011, 29, 1011.

[10] Sun, C. W.; Chen, Y. X.; Liu, T. Y.; Wu, Y.; Fang, T.; Wang, J.; Xing, J. H. Chin. J. Chem. 2012, 30, 1415.

[11] Wang, B. Z.; Cheng, J. G.; Xu, Z. P.; Xu, X. X.; Shao, X. S.; Li, Z. Molecules 2012, 17, 10014.

[12] Zapolskii, V. A.; Fischer, R.; Namyslo, J. C.; Kaufmann, D. E. Bioorg. Med. Chem. 2009, 17, 4206.

[13] Novák, L.; Hornyánszkya, G.; Királya, I.; Rohálya, J.; Kolonitsa, P.; Szántaya, C. Heterocycles 2001, 55, 45.

[14] Tomizawa, M.; Yamamoto, I. Nihon Noyaku Gakkaishi 1993, 18, 91.

[15] Kagabu, S.; Matsuno, H. J. Agric. Food Chem. 1997, 45, 276.

[16] Tomizawa, M.; Zhang, N.; Durkin, K. A.; Olmstead, M. M.; Casida, J. E. Biochemistry 2003, 42, 7819.

[17] Wang, Y. L.; Cheng, J. G.; Qian, X. H.; Li, Z. Bioorg. Med. Chem. 2007, 15, 2624.

[18] Tomizawa, M.; Talley, T. T.; Maltby, D.; Durkin, K. A.; Medzihradszky, K. F.; Burlingame, A. L.; Taylor, P.; Casida, J. E. Proc. Natl. Acad. Sci. U. S. A. 2007, 104, 9075.

[19] Ihara, M.; Okajima, T.; Yamashita, A.; Oda, T.; Hirata, K.; Nishiwaki, H.; Morimoto, T.; Akamatsu, M.; Ashikawa, Y.; Kuroda, S.; Mega, R.; Kuramitsu, S.; Sattelle, D. B.; Matsuda, K. Invert. Neurosci. 2008, 8, 71 .

[20] Shao, X. S.; Li, Z.; Qian, X. H.; Xu, X. Y. J. Agric. Food Chem. 2009, 57, 951 .

[21] Zhang, W. W.; Yang, X. B.; Chen, W. D.; Xu, X. Y.; Li, L.; Zhai, H. B.; Li, Z. J. Agric. Food Chem. 2010, 58, 2741.

[22] Ye, Z. J.; Xia, S.; Shao, X. S.; Cheng, J. G.; Xu, X. Y.; Xu, Z. P.; Li, Z.; Qian, X. H. J. Agric. Food Chem. 2011, 59, 10615.

[23] Shao, X. S.; Xu, Z. P.; Zhao, X. F.; Xu, X. Y.; Tao, L. M.; Li, Z.; Qian, X. H. J. Agric. Food Chem. 2010, 58, 2690.

[24] Tomizawa, M.; Casida, J. E. J. Agric. Food Chem. 2011, 59, 2825.

[25] Tomizawa, M.; Casida, J. E. Acc. Chem. Res. 2009, 42, 260.

[26] Talley, T. T.; Harel, M.; Hibbs, R. E.; Radic, Z.; Tomizawa, M.; Casida, J. E.; Taylor, P. Proc. Natl. Acad. Sci. U. S. A. 2008, 105, 7606. 